\title{
Stem cell factor in the serum of patients with esophageal cancer in relation to its histological types
}

Marta Łukaszewicz-Zając ${ }^{1,2}$, Barbara Mroczko ${ }^{2,3}$, Mirosław Kozłowski ${ }^{4}$, Maciej Szmitkowski ${ }^{1,2}$

${ }^{1}$ Department of Biochemical Diagnostics, Medical University of Bialystok, Bialystok, Poland

2Department of Biochemical Diagnostics, University Hospital in Bialystok, Bialystok, Poland

${ }^{3}$ Department of Neurodegeneration Diagnostics, Medical University of Bialystok, Bialystok, Poland

${ }^{4}$ Department of Thoracic Surgery, Medical University of Bialystok, Bialystok, Poland

Submitted: 23 September 2015

Accepted: 3 November 2015

Arch Med Sci 2017; 13, 6: 1357-1364

DOI: 10.5114/aoms.2016.61695

Copyright $\odot 2016$ Termedia \& Banach

\section{Abstract}

Introduction: Hematopoietic growth factors (HGFs), such as stem cell factor (SCF), may stimulate proliferation and differentiation of hematopoietic progenitor cells. Stem cell factor is also able to affect the growth of malignant tumors, including esophageal cancer (EC). The prognosis of EC patients' survival is still unfavorable. Thus, novel biomarkers are necessary to improve the diagnosis and prognosis of EC patients. The aim of this study was to determine the serum SCF concentrations in EC patients in relation to its histological types and compare these levels with the classical tumor marker - carcinoembryonic antigen (CEA).

Material and methods: The study included 56 EC patients and 65 healthy controls. Serum SCF and CEA concentrations were measured using immunoenzyme assays. Moreover, diagnostic criteria of both proteins tested and the survival of EC patients were assessed.

Results: The serum SCF concentrations were lower in EC patients compared to healthy controls, but the difference was not significant, whereas CEA levels were higher in EC patients than in healthy subjects. The serum SCF concentrations were significantly higher in patients with adenocarcinoma of the esophagus (AC) than in patients with esophageal squamous cell carcinoma (ESCC). Moreover, the diagnostic sensitivity of SCF (88\%) was higher than for CEA (29\%) and increased for combined analysis of SCF with CEA.

Conclusions: Our findings suggest the potential role of serum SCF in the diagnosis of EC patients, especially in combination with the classical tumor marker. However, due to the non-specific nature of SCF, this issue requires further investigations performed on a larger population of EC patients.

Key words: cancer, cytokine, esophageal, marker, stem cell factor.

\section{Introduction}

Esophageal cancer (EC) is an aggressive malignant tumor of the gastrointestinal tract. Esophageal squamous cell carcinoma (ESCC) and adenocarcinoma of the esophagus (AC) are the most common histological types of EC. The pattern of EC has changed in the Western population, and $\mathrm{AC}$ has become the most common type of this malignancy. This may be explained by the increased prevalence of obesity and gastroesopha-

\author{
Corresponding author: \\ Marta Łukaszewicz-Zając PhD \\ Department of \\ Biochemical Diagnostics \\ Medical University \\ of Białystok \\ 15 a Waszyngtona St \\ 15-269 Białystok, Poland \\ Phone: +48-85-7468785 \\ Fax: $+48-85-7468585$ \\ E-mail: \\ marta.lukaszewicz-zajac@ \\ umb.edu.pl
}


geal reflux disease (GERD) in Western countries [1-5]. The prognosis of EC patients' survival is still poor due to late diagnosis, biological features of the tumor and lack of early symptoms of the disease. The routine methods, such as endoscopic ultrasonography or computed tomography (CT) have a limited usefulness in early detection of EC, especially in the diagnosis of microscopic lymph node metastases $[6,7]$. Therefore, other diagnostic and prognostic biomarkers, including readily available biochemical markers of esophageal neoplasms, are necessary to improve the diagnosis of patients with this malignancy.

Hematopoietic growth factors (HGFs) are cytokines which are able to induce proliferation and differentiation of hematopoietic progenitor cells. However, these small peptides might also be in-

Table I. Characteristics of esophageal cancer patients (EC)

\begin{tabular}{|c|c|}
\hline Group tested & $\begin{array}{c}\text { Number of } \\
\text { patients }\end{array}$ \\
\hline \multicolumn{2}{|l|}{ Group: } \\
\hline Esophageal cancer (EC) & 56 \\
\hline Control group & 65 \\
\hline \multicolumn{2}{|l|}{ Gender: } \\
\hline Male & 44 \\
\hline Female & 12 \\
\hline \multicolumn{2}{|l|}{ Type of cancer: } \\
\hline Adenocarcinoma (AC) & 23 \\
\hline Planoepitheliale (ESCC) & 33 \\
\hline \multicolumn{2}{|l|}{ TNM stage: } \\
\hline$I+I \mid$ & 15 \\
\hline III & 30 \\
\hline IV & 11 \\
\hline \multicolumn{2}{|l|}{ Depth of tumor invasion (T factor): } \\
\hline $\mathrm{T} 1+\mathrm{T} 2$ & 12 \\
\hline T3 & 27 \\
\hline $\mathrm{T} 4$ & 17 \\
\hline \multicolumn{2}{|l|}{ Nodal involvement (N factor): } \\
\hline NO & 15 \\
\hline N1 & 41 \\
\hline \multicolumn{2}{|l|}{ Distant metastases ( $M$ factor): } \\
\hline MO & 45 \\
\hline M1 & 11 \\
\hline \multicolumn{2}{|l|}{ Differentiation of tumor: } \\
\hline Well-differentiated - G1 & 11 \\
\hline Moderately differentiated - G2 & 26 \\
\hline Undifferentiated - G3 & 18 \\
\hline
\end{tabular}

volved in the regulation of growth and spread of malignant tumors [8]. Some clinical investigations have indicated that several HGFs might be produced aberrantly in many malignancies [9]. These cytokines may also serve as autocrine or paracrine growth factors [8] as well as indicators of an immune response to the tumors [10]. Additionally, the receptors for selected HGFs have been found on nonhematopoietic tumor cell lines [8]. Oka et al. reported the presence of stem cell factor (SCF) mRNA in human esophageal cancer cell lines [11]. Moreover, changes in the concentrations of SCF were observed in the sera of patients with gastric [12], breast [13], colorectal [14, 15] and pancreatic [16-18] cancer. However, according to our knowledge, this is the first study assessing the serum SCF concentrations in relation to its histological types (ESCC and AC) in comparison to serum levels of the classic tumor marker for EC (CEA). In addition, the association between serum SCF concentrations and clinicopathological tumor characteristics as well as survival of EC patients was evaluated. Moreover, diagnostic criteria, including diagnostic sensitivity and specificity, and predictive value for positive $\left(\mathrm{PV}_{\text {tve }}\right)$ and negative $\left(\mathrm{PV}_{\text {-ve }}\right)$ results for both proteins tested were calculated. The present report is the continuation of our previous investigations, where we assessed the usefulness of selected hematopoietic cytokines in the diagnosis of gastrointestinal malignancies $[12,14,15,18]$.

\section{Material and methods}

\section{Patients}

Our inclusion criteria were as follows: 56 patients with esophageal cancer ( 44 men and 12 women, aged 44-81 years) diagnosed by the Oncology Group and operated on by the Thoracic Surgery Unit of the University Hospital of Bialystok. Among EC patients, 33 subjects suffered from ESCC, while in 23 patients AC was diagnosed. The microscopic examination of material obtained during biopsy and/or surgery as well as barium and chest radiography; endoscopy of the tracheobronchial tree, pharynx, larynx and esophagus; computed tomography as well as magnetic resonance imaging (MRI) examination of the thorax and abdomen were used for the clinical diagnosis of patients with EC. All the tumors were staged in accordance with the tumor-node-metastasis (TNM) classification, proposed by the $5^{\text {th }}$ International Union Against Cancer (UICC) [19]. The control group included 65 healthy volunteers (33 women and 32 men, aged 21-69 years), and they were recruited from hospital volunteers organizations. The characteristics of EC patients are presented in Table I.

All subgroups analyzed in the study (patients with EC, ESCC and AC) were divided into the fol- 
lowing groups: depending on tumor stage $(\mathrm{I}+\mathrm{II}$, III, and IV), depth of tumor invasion (T1 + T2, T3, and $\mathrm{T} 4)$, the presence of lymph node metastasis (NO and N1) and distant metastasis (MO and M1) as well as histological grade of tumor (G1, G2 and G3). The present study was approved by the Local Ethics Committee (R-I-002/442/2010) and all the patients gave informed consent.

\section{Biochemical analyses}

Blood samples from all the patients were drawn before treatment (Sarstedt, Nümbrecht, Germany) and stored at $-80^{\circ} \mathrm{C}$ until analysis.

The serum SCF concentrations were measured using enzyme-linked immunosorbent assay kits (ELISA) (R\&D Systems, Abingdon, UK) according to the manufacturer's instructions. The intra-assay coefficient of variation (CV\%) for SCF is reported by the manufacturer to be $4.0 \%$ at a mean concentration of $1139 \mathrm{pg} / \mathrm{ml}$ with standard deviation $(\mathrm{SD})=5.5 \mathrm{pg} / \mathrm{m}$. Serum CEA concentrations were measured using microparticle enzyme immunoassay kits (MEIA) (Abbott, Chicago, IL, USA). The intra-assay CV\% for CEA as reported by the manufacturer of the assay kits is $4.9 \%$ at a mean concentration of $2.2 \mathrm{ng} / \mathrm{ml}$, $\mathrm{SD}$ of $0.11 \mathrm{ng} / \mathrm{ml}$.

The reference cut-off values for SCF (1285.0 pg/ $\mathrm{ml})$ and CEA $(4.0 \mathrm{ng} / \mathrm{ml})$ concentrations (the $95^{\text {th }}$ percentile) corresponded to the highest accuracy (minimal false-negative and false-positive results) [12, 14-16, 18].

\section{Statistical analysis}

In the preliminary statistical analysis ( $\chi^{2}$ test) the values of SCF and CEA concentrations did not follow a normal distribution, so nonparametric statistical analysis was employed. The comparisons between two groups were performed by the Mann-Whitney U-test. For three or more groups, the one-way ANOVA Kruskal-Wallis test was used. If significant differences were assessed, the post hoc Dwass-Steel-Critchlow-Fligner test was used to determine which groups were different. Data are presented as median and range. The differences were considered to be statistically significant when $p<0.05$. The correlations were determined by the Spearman method, whereas the Kaplan-Meier method was used for calculation of the survival curves. The log-rank test for univariate analyses of survival and the Cox proportional hazards model for multivariate analyses were employed. In addition, diagnostic criteria, such as diagnostic sensitivity and specificity, and predictive value for positive $\left(P V_{+v e}\right)$ and negative $\left(P V_{-v e}\right)$ results of SCF and CEA, were calculated. Statistical analysis was performed using the Statistica
5.1 PL program (StatSoft Inc., Tulsa, OK, USA), and for diagnostic criteria MedCalc statistical software (Mariakerke, Belgium) and Microsoft Office Excel were used.

\section{Results}

The medians and ranges of SCF and classic tumor marker (CEA) concentrations in EC patients and healthy volunteers are presented in Table II. The SCF levels were lower in EC patients in comparison to healthy controls, but the difference was not significant, whereas CEA was found to be statistically significantly higher in EC patients than in healthy subjects (Table II).

If we consider the histological type of EC, the serum concentrations of SCF were significantly higher in patients with AC than in the ESCC subgroup (Table II). Similar results were obtained for CEA levels, but these differences were not statistically significant. Moreover, serum SCF concentrations were higher in the early stage of disease than in stage IV of EC. Opposite results were found for the assessment of CEA concentrations, where CEA concentrations in patients with stage III EC were significantly higher than those in stage I + II tumors (Table II).

In the present study we assessed the SCF and CEA concentrations in relation to clinicopathological parameters of EC, such as depth of tumor invasion, the presence of lymph node and distant metastases as well as histological grade of tumor. Serum SCF concentrations decreased with the depth of tumor invasion ( $T$ factor) and were the lowest in the T4 subgroup, while CEA levels were higher in $\mathrm{T} 4$ patients than in $\mathrm{T} 1+\mathrm{T} 2$ or T3 subgroups. The serum concentrations of SCF were higher in patients without the presence of lymph node (NO) and distance metastases (MO) than in $\mathrm{N} 1$ and M1 subgroups, but these differences were not significant. The concentrations of CEA were significantly higher in EC patients with the presence of lymph node metastases than in the NO subgroup. Moreover, the serum concentrations of SCF were the highest in poorly differentiated tumors (G3), while CEA concentrations were the highest in the G2 subgroup (Table II).

Logistic regression analysis indicated that the serum concentrations of SCF significantly correlated with age $(p=0.002)$ of EC patients, while CEA levels were positively associated with the depth of tumor invasion $(p=0.044)$ and tumor size $(p=0.002)$.

The diagnostic sensitivity of SCF and the classic tumor marker in EC patients is presented in Figure 1 . The frequency of increased SCF concentrations (88\%) was much higher than for CEA (29\%). The combined use of SCF with CEA in EC patients increased their diagnostic sensitivity up to $91 \%$ 
Table II. Serum concentrations of stem cell factor (SCF) and classic tumor marker (CEA) in esophageal cancer (EC) patients

\begin{tabular}{|c|c|c|c|c|c|c|c|}
\hline \multirow[t]{2}{*}{ Variable } & & \multicolumn{3}{|c|}{ SCF [pg/ml] } & \multicolumn{3}{|c|}{ CEA [ng/ml] } \\
\hline & & Median & Range & $P$-value & Median & Range & $P$-value \\
\hline \multirow[t]{2}{*}{ Group tested } & $\begin{array}{l}\text { Esophageal } \\
\text { cancer (EC) } \\
(n=56)\end{array}$ & 1020.1 & $436.2-1688.2$ & 0.971 & 2.4 & $0.4-40.4$ & $<0.001^{*}$ \\
\hline & $\begin{array}{l}\text { Control group } \\
(n=65)\end{array}$ & 1029.0 & $642.0-1812.0$ & & 0.9 & $0.0-2.6$ & \\
\hline \multirow[t]{2}{*}{$\begin{array}{l}\text { Type of } \\
\text { cancer }\end{array}$} & $\begin{array}{l}\text { Adenocarcinoma } \\
(\text { AC) } \\
(n=23)\end{array}$ & 1072.3 & $663.1-1688.3$ & $0.022^{*}$ & 2.5 & $0.4-40.4$ & 0.777 \\
\hline & $\begin{array}{l}\text { Planoepitheliale } \\
\text { (ESCC) } \\
(n=33)\end{array}$ & 971.3 & $436.2-1267.3$ & & 2.3 & $0.4-8.5$ & \\
\hline \multirow[t]{3}{*}{ TNM stage } & $\begin{array}{l}I+I I \\
(n=15)\end{array}$ & 1023.8 & $737.9-1267.3$ & 0.665 & $1.8^{* *}$ & $0.4-3.3$ & $0.020^{*}$ \\
\hline & $\begin{array}{l}\text { III } \\
(n=30)\end{array}$ & 1029.6 & $436.2-1688.2$ & & 2.9 & $0.4-40.4$ & \\
\hline & $\begin{array}{l}\text { IV } \\
(n=11)\end{array}$ & 942.3 & $728.2-1211.1$ & & 2.3 & $0.4-7.8$ & \\
\hline \multirow{3}{*}{$\begin{array}{l}\text { Depth of } \\
\text { tumor } \\
\text { invasion } \\
\text { (T factor) }\end{array}$} & $\begin{array}{l}\mathrm{T} 1+\mathrm{T} 2 \\
(n=12)\end{array}$ & 1032.5 & $737.9-1211.1$ & 0.564 & 1.7 & $0.4-5.0$ & 0.098 \\
\hline & $\begin{array}{l}\text { T3 } \\
(n=27)\end{array}$ & 1016.4 & $685.2-1688.2$ & & 2.5 & $0.8-40.4$ & \\
\hline & $\begin{array}{l}\mathrm{T} 4 \\
(n=17)\end{array}$ & 982.9 & $436.2-1420.0$ & & 3.1 & $0.4-23.8$ & \\
\hline \multirow{2}{*}{$\begin{array}{l}\text { Nodal } \\
\text { involvement } \\
\text { (N factor) }\end{array}$} & $\begin{array}{l}\text { No } \\
(n=15)\end{array}$ & 1023.8 & $803.9-1267.3$ & 0.875 & 1.9 & $0.4-3.3$ & $0.015^{*}$ \\
\hline & $\begin{array}{l}\mathrm{N} 1 \\
(n=41)\end{array}$ & 1016.4 & $436.2-1688.2$ & & 2.7 & $0.4-40.4$ & \\
\hline \multirow{2}{*}{$\begin{array}{l}\text { Distant } \\
\text { metastases } \\
\text { (M factor) }\end{array}$} & $\begin{array}{l}\text { MO } \\
(n=45)\end{array}$ & 1023.8 & $436.2-1688.2$ & 0.433 & 2.5 & $0.4-40.4$ & 0.975 \\
\hline & $\begin{array}{l}M 1 \\
(n=11)\end{array}$ & 942.3 & $728.2-1211.1$ & & 2.3 & $0.4-7.8$ & \\
\hline \multirow[t]{3}{*}{$\begin{array}{l}\text { Differentiation } \\
\text { of tumor }\end{array}$} & $\begin{array}{l}\mathrm{G} 1 \\
(n=11)\end{array}$ & 1010.6 & $686.5-1359.4$ & 0.931 & 1.9 & $0.5-7.8$ & 0.391 \\
\hline & $\begin{array}{l}\mathrm{G} 2 \\
(n=26)\end{array}$ & 1016.4 & $685.2-1688.2$ & & 2.6 & $0.5-40.4$ & \\
\hline & $\begin{array}{l}\text { G3 } \\
(n=18)\end{array}$ & 1059.0 & $436.2-1413.8$ & & 2.4 & $0.4-23.8$ & \\
\hline
\end{tabular}

${ }^{\star}$ Statistically significant when $p<0.05 .{ }^{* \star}$ Statistically significant in post hoc Dwass-Steel-Critchlow-Fligner test in comparison with stage III $(p=0.013)$

(Figure 1). A similar tendency was observed for AC patients, where the highest values of diagnostic sensitivity were found for combined use of SCF with CEA (78\%) (Figure 1). The highest diagnostic specificity was observed for CEA in all analyzed subgroups (EC, ESCC and AC patients). It was likewise in the case of the predictive value for positive $\left(\mathrm{PV}_{+\mathrm{ve}}\right)$ results. In EC patients the predictive value for negative $\left(\mathrm{PV}_{-\mathrm{ve}}\right)$ results of SCF $(56 \%)$ was slightly lower than CEA (62\%), while in the ESCC group PV -ve for SCF (100\%) was higher than for the classical tumor marker (71\%).

The relationship between survival of EC patients and serum concentrations of proteins test- ed was assessed using the Kaplan-Meier method. The univariate log-rank analysis showed that the tumor stage $(p<0.001)$, tumor size $(p=0.012)$, depth of tumor invasion $(p=0.001)$ and presence of distant metastases $(p<0.001)$ were significant factors affecting overall survival. Multivariate regression analysis with Cox's proportional hazards model revealed that only tumor stage $(p=0.020)$, tumor size $(p=0.025)$ and presence of distant metastasis ( $p=0.015$ ) were independent prognostic factors for the survival of EC patients. None of the proteins tested was found to be an independent prognostic factor for EC patients' survival (Figure 2). 


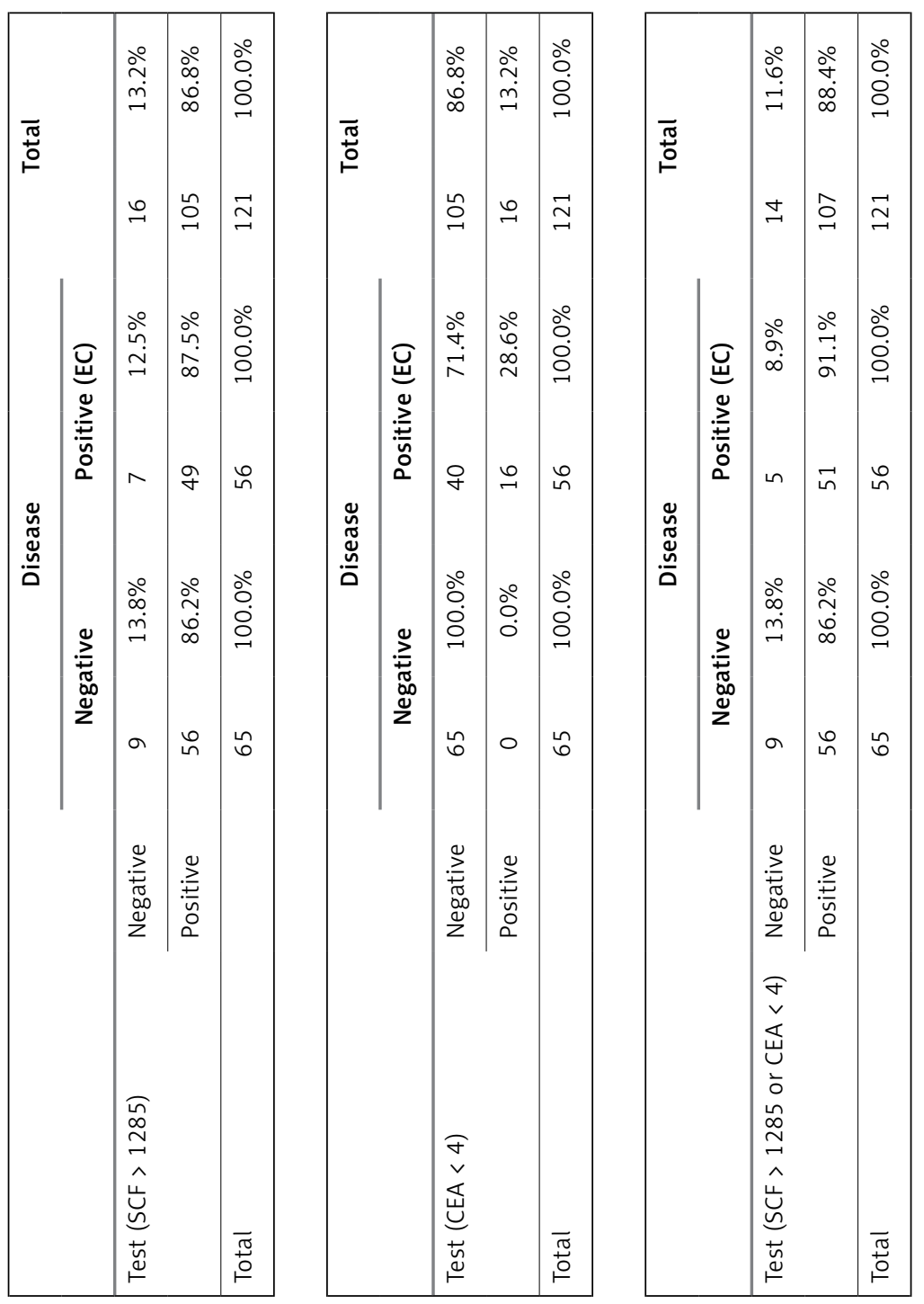

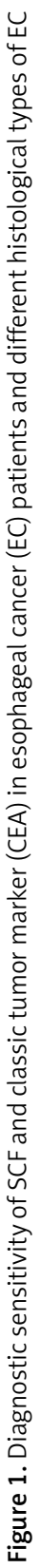




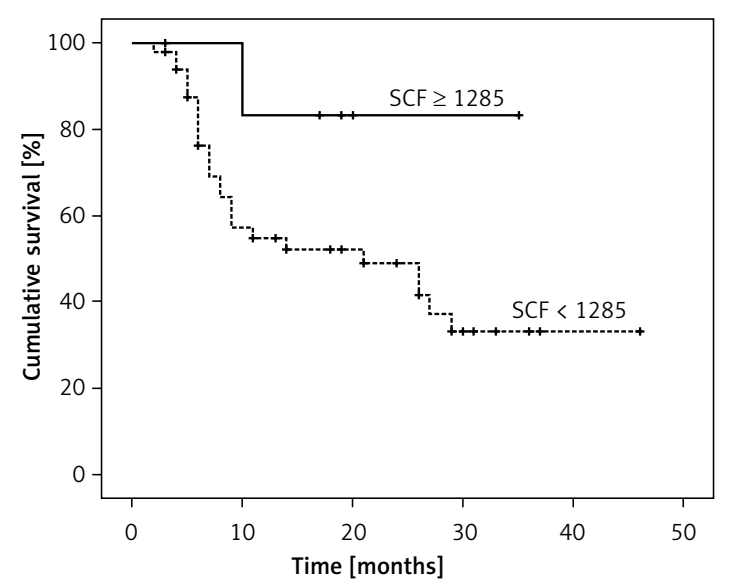

Figure 2. Kaplan-Meier survival analysis for stem cell factor (SCF) concentrations in esophageal cancer patients (EC)

\section{Discussion}

Hematopoietic growth factors, such as SCF, are produced by many neoplasms and might be involved in the regulation of growth and spread of malignant tumors [8]. It was found that several cell lines of malignant tumors are capable of secreting large amounts of HGFs and expressing their receptors $[20,21]$. Stem cell factor, also known as a steel factor, is a multifunctional cytokine that might be involved in tumor development [17, 22]. Recently, increasing evidence has suggested that the SCF/c-kit system is up-regulated in several human malignancies and might play an important role in the angiogenesis, proliferation and invasion of tumor cells [17, 23]. Oka et al. demonstrated the mRNA expression of SCF in human esophageal cancer cell lines [11]. The authors suggested the existence of a complicated cytokine network around esophageal carcinomas that may affect the growth and proliferation of this malignancy [11].

Esophageal cancer is still one of the most aggressive malignancies of the gastrointestinal tract and is characterized by poor prognosis of patients' survival. One of the most commonly used biochemical tumor markers for EC is CEA, but its sensitivity is not satisfactory $[1-5,24,25]$. In the order to improve the clinical diagnosis of patients with this neoplasm, the establishment of novel, easy to perform and readily available biochemical markers has recently been under intensive investigation. According to our knowledge, the present study is the first to report the serum SCF concentrations in patients with EC in relation to different types of this malignancy. Therefore, the aim of our study was to evaluate the clinical value of serum SCF in comparison to the classic tumor marker for EC (CEA) in relation to its histological types. The present paper is a continuation of our previous findings concerning the role of selected HGFs, including SCF as tumor markers for breast [13], colorectal [14, 15], pancreatic [16, 18] and gastric [12] cancer.

In our study, the serum concentrations of SCF were not significantly different between EC patients and healthy controls, whereas the classic tumor marker CEA was found to be statistically significantly higher in EC patients than in healthy subjects. These results are in agreement with our previous findings and the studies of other authors performed on patients with colorectal [14, 15], gastric [12] and pancreatic [16, 18, 26] cancer, where serum SCF concentrations were also lower in cancer patients when compared to a healthy group; however, those differences were statistically significant. Moreover, the serum SCF concentrations were significantly lower in the ESCC subgroup when compared to patients with AC. These results might suggest that the serum levels of this cytokine may be affected by the histological type of EC and confirmed marked differences in both types of EC not only in the biology, but also in the clinico-pathological characteristics of these malignancies. Therefore, the present findings suggested that the measurement of SCF concentrations could be useful in the differentiation between the two most common histological types of EC. Similar results were obtained for CEA levels, but these differences were not statistically significant.

If we consider the TNM classification, the concentrations of SCF were higher in the early stage of disease than in stage IV of EC. A similar tendency was observed in our previous studies concerning the serum SCF levels in patients with colorectal $[14,15]$ and gastric cancer [12], although the differences between serum SCF concentrations and tumor stages were statistically significant only in colorectal cancer patients [15]. Elevated serum concentrations of SCF in patients with early stage EC might be a result of its production by tumor cells in the first step of neoplasm development. Moreover, the alterations in the serum levels of this cytokine could be explained by the changes in the immune system [15]. In addition, the SCF/c-kit system may have a growth-regulating role in the normal tissues, and during malignant transformation this network might be modified [14]. In our current study, the serum SCF concentrations were higher in patients without lymph node and distant metastases than in patients with nodal involvement and distant metastases. Previously, we also revealed the association between serum levels of selected HGFs and clinicopathological tumor parameters; however, those studies were performed on gastric, pancreatic and colorectal patients [12, $14,15,18]$. Our present study suggests the potential role of SCF in the development of EC, especially in the early stage of this malignancy [15]. 
In the present study the percentage of elevated results of SCF was much higher than for CEA and increased for combined use of both proteins tested, similarly as for the AC subgroup. Thus, in order to achieve diagnostic sensitivity, the combined analysis of SCF with the classic tumor marker may be more useful in the diagnosis of EC than measurement of a single marker. Previously, we also calculated the diagnostic sensitivity of SCF in colorectal [15] and pancreatic cancer patients [16]. The combined use of SCF with other classic tumor markers also increased the diagnostic sensitivity in patients with pancreatic [16] and colorectal cancer [15]. The highest diagnostic specificity was observed for CEA concentrations in all analyzed subgroups (EC, ESCC and AC patients), and it was likewise for the $P V_{+v e}$ results. Similar findings were obtained in our previous papers, where the diagnostic specificity of SCF was also lower than for CEA in colorectal cancer patients $[14,15]$. In our study, the $P V_{-v e}$ results of SCF were slightly lower than those of CEA, while in the ESCC group $\mathrm{PV}_{\text {-ve }}$ for SCF (100\%) was higher than for the classic tumor marker (71\%). The highest predictive value for negative results was observed for combined use of both analyzed proteins (SCF and CEA) in EC and AC subgroups. In colorectal cancer patients the $\mathrm{PV}_{-\mathrm{ve}}$ results of SCF was also slightly higher than for the classic tumor marker (CEA) [15].

In conclusion, the present study is a continuation of our previous findings assessing the role of selected HGFs, such as SCF, as potential biomarkers for malignancies of the gastrointestinal tract. We revealed the potential significance of serum SCF in the differentiation between histological types of EC. Moreover, we suggested the potential role of this cytokine in EC development, especially in the early stage of tumor. In addition, the established classic tumor marker CEA showed the well-known high specificity and predictive value for positive results, but very low sensitivity, whereas very high sensitivity was observed for the measurement of SCF concentrations. Therefore, our findings suggest the potential usefulness of SCF in the diagnosis of EC patients, especially in combined analysis with CEA. However, given the non-specific nature of SCF, the diagnostic value of this cytokine may be limited. According to our knowledge, this paper is the first study concerning the SCF concentration in the sera of EC patients in relation to its histological types. However, further investigations need to be continued and performed on a larger population of EC patients.

\section{Acknowledgments}

The present project was supported by the Medical University of Bialystok, Poland. BM is supported by funds from the Leading National Research
Centre (KNOW), Medical University of Bialystok, Poland.

\section{Conflict of interest}

The authors declare no conflict of interest.

\section{References}

1. Siegel R, Naishadham D, Jemal A. Cancer statistics, 2013. CA Cancer J Clin 2013; 63: 11-30.

2. Crew KD, Neugut Al. Epidemiology of upper gastrointestinal malignancies. Semin Oncol 2004; 31: 450-64.

3. Pohl H, Welch HG. The role of overdiagnosis and reclassification in the marked increase of esophageal adenocarcinoma incidence. J Natl Cancer Inst 2005; 97: 142-6.

4. Arnold M, Soerjomataram I, Ferlay J, Forman D. Global incidence of oesophageal cancer by histological subtype in 2012. Gut 2015; 64: 381-7.

5. Holmes RS, Vaughan TL. Epidemiology and pathogenesis of esophageal cancer. Semin Radiat Oncol 2007; 17: 2-9.

6. Merkow RP, Bilimoria KY, McCarter MD, Chow WB, Ko CY, Bentrem DJ. Use of multimodality neoadjuvant therapy for esophageal cancer in the United States: assessment of 987 hospitals. Ann Surg Oncol 2012; 19: 357-64.

7. Tyers M, Mann M. From genomics to proteomics. Nature 2003; 422: 193-7.

8. McDermott RS, Deneux L, Mosseri V, et al. Circulating macrophage colony stimulating factor as a marker of tumour progression. Eur Cytokine Netw 2002; 13: 121-7.

9. Dunlop RJ, Campbell CW. Cytokines and advanced cancer. J Pain Symptom Manage 2000; 20: 214-32.

10. Balkwill F, Mantovani A. Inflammation and cancer: back to Virchow? Lancet 2001; 357: 539-45.

11. Oka M, Hirose K, lizuka N, et al. Cytokine mRNA expression patterns in human esophageal cancer cell lines. J Interferon Cytokine Res 1995; 15: 1005-9.

12. Mroczko B, Wereszczyńska-Siemiatkowska U, Groblewska $M$, et al. The diagnostic value of hematopoietic cytokines measurement in the sera of gastric cancer and gastric ulcer patients. Clin Chim Acta 2006; 374: 165-7.

13. Ławicki S, Mroczko B, Szmitkowski M. Cytokines as tumor markers in breast cancer. Postep Hig Med Dosw 2003; 57: 455-63.

14. Mroczko B, Szmitkowski M, Wereszczyńska-Siemiatkowska U, Okulczyk B. Stem cell factor (SCF) and interleukin 3 (IL-3) in the sera of patients with colorectal cancer. Dig Dis Sci 2005; 50: 1019-24.

15. Mroczko B, Szmitkowski M, Wereszczyńska-Siemiatkowska U, Okulczyk B, Kedra B. Pretreatment serum levels of hematopoietic cytokines in patients with colorectal adenomas and cancer. Int J Colorectal Dis 2007; 22: 33-8.

16. Mroczko B, Szmitkowski M, Wereszczynska-Siemiatkowska U, Jurkowska G. Stem cell factor and macrophage-colony stimulating factor in patients with pancreatic cancer. Clin Chem Lab Med 2004; 42: 256-60.

17. Gao C, Li S, Zhao T, et al. SCF, Regulated by HIF-1alpha, promotes pancreatic ductal adenocarcinoma cell progression. PLoS One 2015; 10: e0121338.

18. Mroczko B, Szmitkowski M, Wereszczyńska-Siemiatkowska U, Jurkowska G. Hematopoietic cytokines in the sera of patients with pancreatic cancer. Clin Chem Lab Med 2005; 43: 146-50.

19. Jass JR, Sobin LH. WHO International Histological Classification of Tumors. Histological typing of intestinal tumors. Springer-Verlag, New York 1989. 
20. Calatayud S, Warner TD, Breese EJ, Mitchell JA. Modulation by colony stimulating factors of human epithelial colon cancer cell apoptosis. Cytokine 2002; 20: 163-7.

21. Esposito I, Kleeff J, Bischoff SC, et al. The stem cell factor-c-kit system and mast cells in human pancreatic cancer. Lab Invest 2002; 82: 1481-92.

22. Gu Y, Runyan C, Shoemaker A, Surani MA, Wylie C. Membrane-bound steel factor maintains a high local concentration for mouse primordial germ cell motility, and defines the region of their migration. PLoS One 2011; 6: e25984.

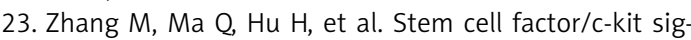
naling enhances invasion of pancreatic cancer cells via HIF-1alpha under normoxic condition. Cancer Lett 2011; 303: 108-17.

24. Zhao H, Zhao L, Zhou Z, Wu Y. The roles of connective tissue growth factor in the development of anastomotic esophageal strictures. Arch Med Sci 2015; 11: 770-8.

25. Motoyama S, Miura M, Hinai Y, et al. CRP genetic polymorphism is associated with lymph node metastasis in thoracic esophageal squamous cell cancer. Ann Surg Oncol 2009; 16: 2479-85.

26. Vasiliades G, Kopanakis N, Vasiloglou M, et al. Role of the hematopoietic cytokines SCF, IL-3, GM-CSF and M-CSF in the diagnosis of pancreatic and ampullary cancer. Int J Biol Markers 2012; 27: e186-94. 